\title{
Non-Invasive Localization of Atrial Flutter Circuit Using Recurrence Quantification Analysis and Machine Learning
}

\author{
Muhammad Haziq Kamarul Azman ${ }^{1}$, Olivier Meste ${ }^{1}$, Decebal G. Latcu ${ }^{3}$, Kushsairy Kadir ${ }^{2}$ \\ ${ }^{1}$ Université Côte d'Azur, CNRS, I3S, France \\ ${ }^{2}$ Universiti Kuala Lumpur, Malaysia \\ ${ }^{3}$ Centre Hospitalier Princesse Grace, Monaco
}

\begin{abstract}
Atrial flutter presents quasi-periodic atrial activity due to circular depolarization. Given the different structure of right and left atria, spatiotemporal variability should be different. This was analyzed using recurrence quantification analysis. Autocorrelation signals were estimated from the unthresholded recurrence plot, calculated with a properly processed ECG to remove variability related to external sources (noise, respiratory motion, $T$ wave overlap). Simple features were considered from the autocorrelation that attempts to describe the atrial activity in terms of range of recurrence and periodicity. Linear classification using support vector machines and logistic regression both allowed good classification performance (max accuracy 0.8 for both). Feature selection showed that right and left AFL have significantly different cycle lengths (right vs. left: $230.63 \mathrm{~ms} v \mathrm{~s} .206 .50 \mathrm{~ms}, p<0.01$ ).
\end{abstract}

\section{Introduction}

The quasi-periodic atrial activity (AA) observed on the electrocardiogram (ECG) during atrial flutter (AFL) is caused by a rotating circular depolarization of the atrium. It has been shown that beat-to-beat variability of the flutter or $\mathrm{F}$ waves, quantified using vectorcardiographic parameters, allowed localization of right or left atrial circuit [1]. Different variability was observed for right and left localization, inducing a hypothesis of varying circuit stability.

With a beat-to-beat approach, instantaneous spatiotemporal information is not preserved, which may contain information about AA. In addition, both atria are known to be remarkably different in structure. The right atrium contains many large and well-defined cardiac fibers and is relatively thin, whereas the left atrium is thick and multilayered [2]. It is expected that spatiotemporal variability would be different.

The use of recurrence quantification analysis (RQA) has been highlighted for spatiotemporal analysis and charac- terization of atrial fibrillation (AF) activation propagation $[3,4]$. Of particular interest, atrial fibrillation recurrence behavior was characterized, and was shown to be different for recurring and non-recurring persistent $\mathrm{AF}$.

In this paper, RQA is employed in order to study the spatiotemporal variability related to the circular propagation of AFL activation in a non-invasive fashion. Several features are extracted from the computed recurrence signal and serves as features for classification of circuit localization. Machine learning techniques are considered in order to obtain practical classifiers as well as to understand the reason why right and left AFL are different by employing feature selection.

\section{Methodology}

\subsection{Dataset and Preprocessing}

54 ECG recordings of AFL were used, acquired using a device (Boston Scientific, USA) at $f_{s}=2000 \mathrm{~Hz}$, during catheter ablation operation at Centre Hospitalier Princesse Grace, Monaco. A finite-impulse response notch filter at $50 \mathrm{~Hz}$ was applied to these signals to remove powerline interference, as well as high- and low-pass filters to keep the band between $[0.5 ; 70] \mathrm{Hz}$. Records with missing leads, low $\mathrm{F}$ wave amplitudes, low atrioventricular block ratio $(<2: 1)$ and low $\mathrm{F}$ wave counts were excluded from the study. In total, there were 30 recordings with right circuit localization and 24 with left localization.

Extraction of atrial activity is crucial for RQA. However, in AFL the high synchronicity between atrial and ventricular activity renders most extraction methods inefficient due to spectral overlap or source dependence. Segmentation of each $\mathrm{F}$ waves is preferred as an alternative to extract atrial activity. Since the essential activity is located fully within the $\mathrm{F}$ wave duration, the remaining portion of signals are theoretically not needed.

$F$ waves from each recording were detected using a method developed previously [5]. Two set of F waves, 
overlapped and not overlapped with $\mathrm{T}$ waves, were segmented. All F waves were synchronized, transformed into VCG and removed of respiratory motion using a technique described previously [1]. Overlapped F waves were corrected using the procedure detailed in a previous study [6].

The mean of each segmented $\mathrm{F}$ waves are removed for all leads. Finally, a new signal, different that the original recording, was made by replacing each wave at the same time index where it was originally located. The rest of the signal not containing any $\mathrm{F}$ waves are padded with zeros. This new signal represents the atrial signal free from any external variability (see 1 , top panel).

\subsection{Calculation of Recurrence Features}

Recurrence quantification analysis (RQA) is a nonlinear technique that aims to quantify the properties of a dynamic, often oscillatory system by comparison of a state space vector $\mathbf{x}(i)$ of dimension $K$ at one instant with another delayed instant $\mathbf{x}(i-\tau)$. In ECG analysis $K$ represents the number of leads available, and $\mathbf{x}(i)$ the ECG voltage at the discrete sample $i$. The state vector is then the multidimensional atrial dipole, and the ECG traces, the trajectory map of the dipole. Due to the circular depolarization, the trajectory resembles a loop.

The unthresholded recurrence plot (URP) $\mathbf{R}$ is a symmetric 2D graphical plot representing a similarity measure $D$ of two states at different delay instants, with the measure being real-valued. This is written as:

$$
R(i, j)=D(\mathbf{x}(i), \mathbf{x}(j))
$$

One intuitive similarity measure that capture spatiotemporal propagation of AA is the normalized dot product:

$$
D(\mathbf{x}(i), \mathbf{x}(j))=\frac{\mathbf{x}(i)^{\mathrm{T}} \mathbf{x}(j)}{\|\mathbf{x}(i)\|\|\mathbf{x}(j)\|}
$$

which is related to the cosine of the angle between the two vectors. This definition of recurrence captures not only the similarity of two very close state vectors, but it also captures similarity when the two vectors are in opposing directions. This was used successfully in the case of AF to characterize its spatiotemporal behavior [3].

A sample URP can be seen in 1 (middle panel) calculated from a 5-second sample of the post-processed AFL signal(top panel). Due to the zero-padding of the postprocessed signal, the URP contains many undefined recurrence values (white area). When defined, the entries in the main diagonal are always 1 , suggesting perfect match. Moving outwards from the main diagonal, repetitive patterns can be observed in intervals.

To limit non-stationarity, the URP was processed in segments of fixed size. A segment starts at the main diagonal, and progresses along the diagonal up to a length $I$. The segment width spans from the main diagonal towards the edge of the plot (i.e. increasing $j$ ) for up to a width $J$. The delay variable can be introduced by $\tau=j-i \Leftrightarrow j=i+\tau$. A segment can be described by the equation:

$$
R_{s}(i, \tau)=R(i, i+\tau), i \in[(s-1) I ; s I]
$$

Highlights of segments can be found in Figure 1. The choice of $I$ and $J$ determines the properties of the segment, and should be considered as parameters. However, it can be reasoned in the context of AFL, that stationarity can be guaranteed for long periods of time due to the regularity of F wave manifestation. For this paper, $I$ is set to 2000 samples (1 second), and $J$ to 4000 samples (2 seconds). Note that $\tau \in[0 ; J]$. The value of $J$ assures that $>5$ AFL cycles can be captured in the segment.

To reduce the variance of the recurrence estimate, an average can be taken for each segment along $i$ :

$$
\bar{D}_{s}(\tau)=\frac{1}{I} \sum_{i=(s-1) I}^{s I} D_{s}(i, \tau)
$$

This amounts to an autocorrelation, assuming stationarity within the segment. In practive, undefined recurrence values were discarded, and for each $\tau$, the average only considers defined values (i.e. $I$ is not fixed), to avoid bias in the calculation. An example of this is shown in Figure 1 (bottom panels). It is clear that AFL has a quasi-periodic recurrence behaviour, as shown by the high and repetitive autocorrelation peaks.

To quantify this behavior, two parameters were considered. The first parameter $S_{1}$ relates to the range of spatial propagation. This is given by the peak-to-peak amplitude of the autocorrelation, taken from one maximum to the next minimum (Figure 1, bottom panel, in green). The second parameter $S_{2}$ relates to the periodicity of propagation, and is essentially an estimate to the AFL cycle length. This is calculated by the peak-to-peak interval of the available maximums. The two parameters are calculated for all available points in the signal, hence making a series of values.

Calculation of these parameters can be done for each segment. However, the existence of long periods of undefined values can be troublesome, since long segments of discontinuity is present. In order to combat this problem, the autocorrelations were aggregated by calculating their median value:

$$
\tilde{D}=\operatorname{median}_{s=1}^{S} D_{s}
$$

The result is a single continuous autocorrelation function. In some patients, the function $\tilde{D}$ was unfortunately still discontinuous due to prolonged undefined periods. This did not hinder the calculation of the parameters, though. 

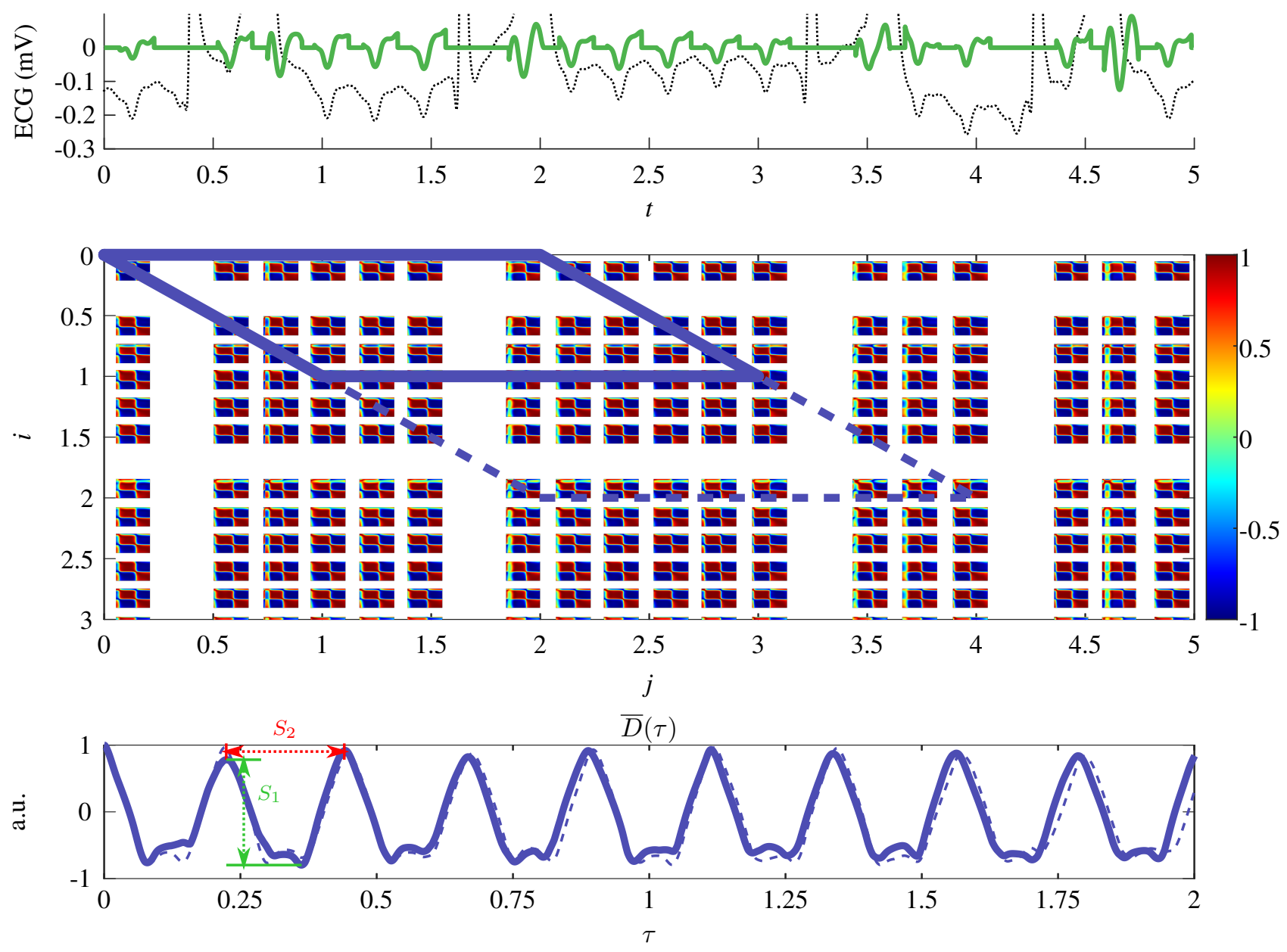

Figure 1. Top panel: 5-second sample ECG (lead $\mathrm{V}_{1}$ ). Thin broken line indicates the post-filtered ECG, whereas thick green line indicates the ECG after all processing steps. Middle panel: unthresholded recurrence plot calculated from the new ECG. Bottom panel: autocorrelation functions derived from their respective plots by averaging of single segments. First segment is shown (thick line) along with the subsequent segment (thin broken line). See Section 2.2 for details.

From the two parameters, several features were calculated, that aim to capture spatiotemporal variability in AFL: the 1) mean, 2) standard deviation, 3) variance, 4) skewness, and 5) kurtosis of the two series. The features are in fact statistical moments, and characterizes the properties of the series distribution. In total, there were 10 features considered.

\subsection{Learning Protocol}

The set of data and features were used as learning inputs to the linear support vector machine (SVM) and logistic regression (LOG) classifiers. Due to the small sample size, no validation schemes were considered. In order to evaluate the relevance of the feature, a wrapper approach was employed by evaluating all possible combinations of fea- tures from 1 to 10 . Relevance of the feature was determined by counting the participation of each feature in the combinations that produced the highest accuracy, from a combination length of 1 to 10 , and summing the participation score.

\section{Results and Discussion}

The result of classification is shown in Figure 2. As can be seen, recurrence quantification gave features that are discriminating with respect to AFL localization. Good performance can be achieved, as seen by accuracies $>0.6$, and increases as more features are added. Maximum performance was obtained by all classifiers at 6 combinations of features (accuracy $=0.8$, sensitivity and specificity $=$ $(0.67,0.90)$ and $(0.90,0.67)$ for LOG and SVM classi- 


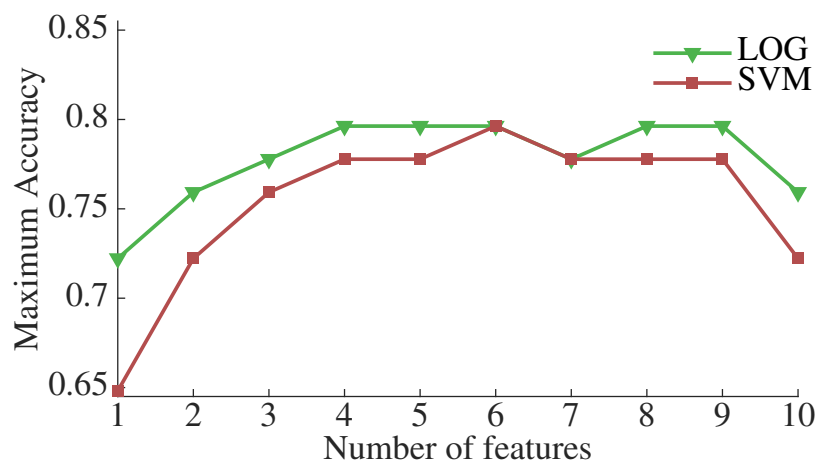

Figure 2. Maximum classifier accuracy of the considered classifiers.

fier respectively). This was obtained using linear classifiers and with a small amount of features, suggesting that overfitting was avoided. The 6 features used were $\left[\begin{array}{llllll}F_{1} & F_{2} & F_{3} & F_{6} & F_{7} & F_{9}\end{array}\right]$ for LOG, and $\left[\begin{array}{llllll}F_{1} & F_{2} & F_{5} & F_{6} & F_{7} & F_{9}\end{array}\right]$ for SVM (refer to Table 1 for feature indices).

Table 1. Feature Scores

\begin{tabular}{r|r|c|c}
\hline \multicolumn{1}{|c}{ Index } & Feature & LOG & SVM \\
\hline$F_{1}$ & $\operatorname{Mean}\left(S_{1}\right)$ & 8 & 6 \\
$F_{2}$ & $\operatorname{Stdev}\left(S_{1}\right)$ & 7 & 7 \\
$F_{3}$ & $\operatorname{Var}\left(S_{1}\right)$ & 7 & 8 \\
$F_{4}$ & $\operatorname{Skewness}\left(S_{1}\right)$ & 4 & 1 \\
$F_{5}$ & $\operatorname{Kurtosis}\left(S_{1}\right)$ & 4 & 7 \\
$F_{6}$ & $\operatorname{Mean}\left(S_{2}\right)$ & $\mathbf{1 0}$ & $\mathbf{1 0}$ \\
$F_{7}$ & $\operatorname{Stdev}\left(S_{2}\right)$ & 7 & 6 \\
$F_{8}$ & $\operatorname{Var}\left(S_{2}\right)$ & 5 & 5 \\
$F_{9}$ & $\operatorname{Skewness}\left(S_{2}\right)$ & 7 & 9 \\
$F_{10}$ & $\operatorname{Kurtosis}\left(S_{2}\right)$ & 4 & 6 \\
\hline \hline
\end{tabular}

Further analysis of the relevant features was performed by counting the score of each feature: if the feature was involved in any combinations of length $l$ that produced the maximum accuracy, then it is assigned a score of 1 , else 0 . The total score is the sum of scores from combination length 1 to 10 . The largest possible score is then 10 , and lowest 0 .

Table 1 shows the feature score for each classifier. The highest participation was found to be of the feature $F_{6}$. This suggests that this feature presents a significant contribution in determining right or left localization. Interpretation of the feature indicates that right flutter have significantly larger cycle lengths (mean $230.63 \mathrm{~ms}$ ) than left flutter (mean $206.50 \mathrm{~ms} ; p<0.01$, Mann-Whitney $U$ test). However, this does not conclude on whether if AA propagation was faster in left AFL or that the circuit was larger. Further investigation is required to clarify this matter.

\section{Conclusion}

It has been shown that the ensemble of processing steps were able to extract meaningful characteristic parameters of the AFL AA propagation, and allowed localization to be performed with a good degree of performance. Recurrence quantification analysis allowed access to spatiotemporal characteristics of AFL, which was shown to be different for each localization. Inspection of the features showed that different AFL localization produced significantly different propagation cycle lengths.

\section{References}

[1] Kamarul Azman MH, Meste O, Kadir K, Latcu DG. Localizing atrial flutter circuit using variability in the vectorcardiographic loop parameters. In Computing in Cardiology, volume 45. 2018; 4.

[2] Wang K, Ho SY, Gibson DG, Anderson RH. Architecture of atrial musculature in humans. Heart June 1995;73(6):559565.

[3] Meste O, Zeemering S, Lankveld T, Schotten U, Crijns H, Peeters R, Bonizzi P. Noninvasive recurrence quantification analysis predicts atrial fibrillation recurrence in persistent patients undergoing electrical cardioversion. In Computing in Cardiology, volume 43. 2016; 677-680.

[4] Bonizzi P, Zeemering S, Karel J, Azman MH, Lankveld T, Schotten U, Crijns H, Peeters R, Meste O. Noninvasive characterisation of short-and long-term recurrence of atrial signals during persistent atrial fibrillation. In Computing in Cardiology, volume 44. 2017; .

[5] Kamarul Azman MH, Meste O, Kadir K. Detecting flutter waves in the electrocardiogram using generalized likelihood ratio test. In Computing in Cardiology, volume 45. sep 2018; 4.

[6] Kamarul Azman MH, Meste O, Kadir K, Latcu DG. Estimation and removal of $t$ wave component in atrial flutter ecg to aid non-invasive localization of ectopic source. In Computing in Cardiology, volume 44. 2017; 4.

Address for correspondence:

Muhammad Haziq Kamarul Azman

Room 416

Universiti Kuala Lumpur, British Malaysian Institute

Batu 8, Jalan Sungai Pusu

53100 Selangor, Malaysia

mhaziq@unikl.edu.my 